\title{
Early antiretroviral therapy-treated perinatally HIV-infected seronegative children demonstrate distinct long-term persistence of HIV-specific T-cell and B-cell memory
}

\author{
Nicola Cotugno ${ }^{a, b, *}$, Elena Morrocchi ${ }^{a, b, *}$, Stefano Rinaldi ${ }^{c}$, \\ Salvatore Rocca ${ }^{a}$, Ilaria Pepponi ${ }^{a}$, Silvia di Cesare ${ }^{a}$, \\ Stefania Bernardi ${ }^{a}$, Paola Zangari ${ }^{a}$, Suresh Pallikkuth ${ }^{d}$, \\ Lesley de Armas ${ }^{\mathrm{d}}$, Ofer Levy ${ }^{\mathrm{e}, \mathrm{f}, \mathrm{g}}$, Paolo Rossi ${ }^{\mathrm{a}, \mathrm{b}}$, Savita Pahwa ${ }^{\mathrm{d}}$, \\ Paolo Palma ${ }^{a}$, on behalf of the EPIICAL Consortium ${ }^{\dagger}$
}

Objective: To investigate long-term persistence of HIV-specific lymphocyte immunity in perinatally HIV-infected children treated within the first year of life.

Design: Twenty perinatally HIV-infected children who received ART therapy within the first year of life (early treated) and with stable viral control ( $>5$ years) were grouped according to their serological response to HIV.

Methods: Western blot analysis and ELISA defined 14 HIV-seropositive and six seronegative patients. Frequencies of gp140-specific T-cell and B-cell, and T-cell cytokine production were quantified by flow cytometry in both seronegatives and seropositives. Transcriptional signatures in purified gp140-specific B-cell subsets, in response to invitro stimulation with HIV peptides was evaluated by multiplex RT-PCR.

Results: Gp140-specific T cells and B cells persist at similar levels in both groups. A higher production of IL-21 in gp140-specific T cells was found in seropositives vs. seronegatives $(P=0.003)$. Gene expression in switched IgM-IgD- gp140-specific memory B cells after stimulation with HIV peptides in vitro demonstrated a differential expression of genes involved in signal transduction and activation after BCR/TLR triggering and B-cell activation. Genes relating to antibody production (PRDM1) and T-B cognate stimulation (CXCR4, IL21R) were differentially induced after in-vitro stimulation in seronegatives vs. seropositives suggesting a truncated process of B-cell maturation.

Conclusion: HIV-specific memory B and T cells persist in early treated regardless their serological status. Seronegatives and seropositives are distinguished by gp140-specific T-cell function and by distinct transcriptional signatures of gp140-specific B cells after in-vitro stimulation, presumably because of a different antigen exposure. Such qualitative insights may inform future immunotherapeutic interventions.

Copyright @ 2020 The Author(s). Published by Wolters Kluwer Health, Inc.

AIDS 2020, 34:669-680

\footnotetext{
${ }^{a}$ Research Unit of Congenital and Perinatal Infections, Bambino Gesù Childrens' Hospital, IRCCS, bepartment of Systems Medicine, Chair of Paediatrics, University of Rome 'Tor Vergata', Rome, Italy, 'Department of Microbiology and Immunology, University of Miami Miller School of Medicine, ${ }^{\mathrm{d}}$ Department of Microbiology and Immunology, Miami Center for AIDS Research

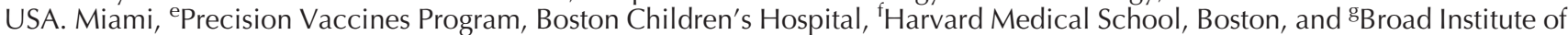
MIT \& Harvard, Cambridge, Massachusetts, USA.

Correspondence to Paolo Palma, MD, PhD, Children's Hospital 'Bambino Gesù' Childrens' Hospital, IRCCS, Piazza Sant'Onofrio 4 , Rome, Italy.

Tel: +39 668593649 3080; e-mail: paolo.palma@opbg.net

* Nicola Cotugno and Elena Morrocchi contributed equally to this work.

${ }^{\dagger}$ A list of members of the EPIICAL Consortium is included in the Acknowledgements.

Received: 20 August 2019; revised: 10 December 2019; accepted: 14 December 2019.
} 


\section{Keywords: early treated children, gene expression, HIV-specific B cells, HIV- specific cell immunity, HIV-specific T cells, pediatric HIV, seronegative early treated children}

\section{Introduction}

Strong evidence indicates that antiretroviral treatment (ART) should be initiated in HIV infected infants early in the first year of life in order to reduce morbidity and mortality [1]. Early control of HIV-1 replication within the first months of life was accompanied by preservation of $\mathrm{CD} 4^{+}$T-cell counts, absence of circulating activated $\mathrm{T}$ cells, and normal immune function [2]. However, these early treatment studies also identified a lack of circulating HIV-1-specific antibodies and T-cell responses as a hallmark of early suppression of HIV-1 replication [2,3]. Several research groups have also found that a consistent proportion of these patients do not display HIV-specific responses [4,5]. The percentage of HIV-infected children who are HIV seronegative has been shown to range between 36 and $46 \%$ of HIV-infected children treated within the first year of life $[3,5,6]$. This condition of seronegativity persists over time after long-term follow-up. A number of studies showed that the absence of HIV antibodies indicates a smaller size of HIV reservoir $[4,5,7]$ making these seronegative children ideal candidates for immune intervention studies towards HIV remission $[8,9]$.

On the other hand, however, it is not known whether the absence of HIV-specific antibodies could represent a limitation during periods of viral re-exposure because of poor ART adherence, or in the perspective of cure strategies.

In this controversial scenario, two main scientific questions arise: if HIV-specific cells persist despite HIV serostatus, and whether, if present, these cells act differentially according to the patient serostatus once they re-encounter the virus [10].

Herein, we explored these two questions in a group of seronegative patients in comparison to seropositive patients treated with ART within the first year of life.

\section{Methods}

\section{Study participants}

Study participants were enrolled at Bambino Gesù Children's Hospital between December 2016 and June 2017. Only patients who started ARTwithin 1 year of life with durable viral control (at least 2 years) were considered eligible. Plasma samples were tested to define HIV-1 serostatus by fourth generation Chemiluminescent
Microparticle Immune Assay (CMIA) (Abbott Architect $\mathrm{HIV} \mathrm{Ag/Ab} \mathrm{Combo} \mathrm{Assay)} \mathrm{and} \mathrm{western} \mathrm{blot} \mathrm{analysis}$ (WB) (Medical Systems, Italy). Western blot was considered positive when at least one antigen gave a positive result. Timing of blood draw and design of the study has been visualized in Supplementary Figure 1, http://links.lww.com/QAD/B647.

\section{Ethics statement}

Bambino Gesù Children's Hospital review board approved the study and written informed consent was obtained from all participants or their legal guardians.

\section{B-cell and T-cell stimulation}

Cryopreserved PBMCs were thawed and resuspended in R10 medium [RPMI 1640 and 10\% fetal bovine serum (FBS) supplemented with 1\% Pen/Strep and L-Glu. For B cell stimulation, $3 \times 10^{6} \mathrm{cells} / \mathrm{ml}$ were incubated for $16 \mathrm{~h}$ at $37^{\circ} \mathrm{C}$ and $5 \% \mathrm{CO}_{2}$ with a mix of three different pools of ENV peptides $(100 \mu \mathrm{g} / \mathrm{ml}$, EURIPRED) and CD40L (250 ng/ml, ENZO Life Sciences, Pero, Italy.). For T-cell stimulation, $5 \times 10^{6}$ PBMCs were rested over night at $37^{\circ} \mathrm{C}, 5 \% \mathrm{CO}_{2}$ in $\mathrm{R} 10$ medium prior to $12 \mathrm{~h}$ incubation with $2 \mu \mathrm{g} / \mathrm{ml}$ gp140 (kindly provided by Dr Kalyanaraman, ABL, Maryland, USA), $1 \mu \mathrm{g} / \mathrm{ml}$ of staphylococcal enterotoxins B (SEB, List Biological Laboratories, Inc., Campbell, California, USA) or medium (negative control) with the addition of $1 \mu \mathrm{g} / \mathrm{ml}$ of anti-CD28 (BD Biosciences, San Jose, California, USA).

\section{Flow cytometry, cell sorting, RNA extraction and reverse transcription}

Following specific stimulation, PBMCs were stained for B-cell phenotype with the following monoclonal antibodies (mAbs, from $\mathrm{BD}$ Biosciences): $\mathrm{CD}^{+}$, $\mathrm{CD}_{10}{ }^{+}, \mathrm{CD}^{+}{ }^{+}$(BV510), $\mathrm{CD} 19^{+}$(APC-R700), $\mathrm{CD}_{21}{ }^{+}$(APC), $\mathrm{CD}^{+} 7^{+}$(FITC), $\mathrm{CD}^{+} 8^{+}$(PE-Cy7), $\mathrm{CD}^{+}{ }^{+}$(PErCP-Cy5.5), $\mathrm{CD}^{+} \quad$ (APC-H7) $\mathrm{IgD}$ (BV421), IgM (PE-CF594), IgG (BV605, HIV-1 envelope trimeric gp140 protein (gp140-R-Phycoerythrin (-R-PE)) and Live/Dead (BV510; BD Horizon, Franklin Lakes, New Jersey, USA). T-cell phenotype using the following mAbs: $\mathrm{CD}^{+}$(BUV496), CD4 (APC-Cy7), CD27 ${ }^{+}$(BV480), CXCR5 (Alexa647), IFN $\gamma$ (PE-Cy7), IL-2 (BV711) and TNF $\alpha$ (FITC) from BD Biosciences, CD8 (PerCP) and CD40L (BV605) from BD BioLegend, IL-21 (PE, eBioscience), CD45RO (PE-TexasRed, Beckman Coulter, Irving, Texas, USA) and Live/Dead Fixable Blue Dead Stain Kit (ThermoFisher Scientific, Rome, Italy). The gp140 protein was obtained from European Research Infrastructures for 
Poverty Related Disease (EURIPRED) and LightningLink R-PE Conjugation Kit (Innova Biosciences) was used to obtain a labelled gp140-R-PE. Cells were acquired on a BD LSRFortessa and analyzed by FlowJo v10.0.8r1 (Treestar, Inc., Ashland, Oregon, USA). Sorting was performed as shown in Supplementary Figure 2, http://links.lww.com/QAD/B648 using FACSAriaII (BD Biosciences); sorted cells were collected in prelabelled tubes containing reverse transcription buffer for consequent PCR prior to Fluidigm [11].

\section{Multiplexed reverse transcription-PCR (Fluidigm)}

Amplified cDNAs were loaded onto 96.96 integrated fluidic circuit chip (Fluidigm; Biomark, South San Francisco, California, USA), according to manufacturer's instructions. Previously validated Taqman probes (Supplementary Table 1, http://links.lww.com/QAD/B652), polymerases and primers were mixed with samples as described earlier [11]. Cycle threshold $(\mathrm{Ct})$ values were normalized as already described [12].

\section{Statistical analysis}

Unpaired $t$-test or Mann-Whitney U-test were performed to analyze normally and not-normally distributed FACS data; paired Wilcoxon test was used to compare different conditions among the same study participant. Boolean analysis and figures were produced using Simplified Presentation of Incredibly Complex Evaluations (SPICE, distributed by the National Institute of Allergy and Infectious Diseases, NIH (http://exon.niaid. nih.gov/ spice). Statistical tests were performed with Prism 6.0 (GraphPad, Prism). Gene expression data analysis and outlier identification were executed using SingulaR loaded on R. ANOVA was used to identify DEGs and DIGs. FC was determined using mean Expression threshold (Et) values and volcano plots were generated in Prism 6.0. Statistical differences in gene expression between unstimulated and stimulated samples were determined by Wilcoxon matched paired tests. Pearson or Spearman correlation tests were used, respectively for normally or not-normally distributed data. Correlation matrix analysis was performed on ggplot2 package loaded on R.

\section{Results}

\section{Limited exposure to HIV replication after perinatal infection and early treatment initiation correlated with lower HIV antibody response}

ART-treated HIV-perinatally infected children who started therapy within the first year of life $(N=20)$ with durable viral control (see Table 1) were investigated for their HIV antibody response to determine their serostatus. Six participants $(6 / 20,30 \%)$ were seronegatives as defined by complete absence of antibodies against all antigens tested, whereas seropositives were defined as positive for at least one antigen in either ELISA or western blot analysis (10 HIV antigens tested) (Supplementary Figure 3A, http://links.lww.com/QAD/B649). Patients' characteristics are shown in Table 1 . No differences in terms of age, time on suppressive ART, serum measurements of $\operatorname{IgG}, \operatorname{IgM}$ and $\operatorname{IgA}, \mathrm{CD}^{+}$frequency and count were found between seronegatives and seropositives (Table 1). Timing of ART initiation, time to virus suppression (time from ART start to HIV RNA $<50$ copies/ml), duration of plasma viremia (time from birth and HIV-RNA $<50$ copies $/ \mathrm{ml}$ ), and area under the curve of HIV RNA, as revealed by longitudinal collection of viral load analysis (Supplementary Figure 3B and C, http://links.lww.com/ $\mathrm{QAD} / \mathrm{B} 649$ and Table 1) were lower in seronegative

Table 1. Patients' characteristics.

\begin{tabular}{|c|c|c|c|}
\hline & Seronegative & Seropositive & $P$ value \\
\hline Participants, $n$ (female) & $6(5)$ & $14(8)$ & n.s. \\
\hline Age (years), mean (SEM) & $9.16(2.1)$ & $8.06(1.5)$ & n.s. \\
\hline Time of ART start (weeks) mean (SEM) & $7.33(2.6)$ & $22.21(3.9)$ & 0.014 \\
\hline Time on suppressive ART (years), mean (SEM) & $8.43(1.9)$ & $6.78(1.4)$ & n.s. \\
\hline Time to viral suppression (week) Mean (SEM) & $10.17(2.4)$ & $32.21(7.3)$ & 0.024 \\
\hline Duration of plasma viremia (week), mean (SEM) & $21.62(2.75)$ & $62.31(7.55)$ & 0.0001 \\
\hline AUC HIV RNA, area $(95 \% \mathrm{CI})$ & $2.04 \cdot 106(85915-223962)$ & $1.06 \cdot 107(11370-60077)$ & 0.02 \\
\hline Peak viremia, mean (SEM) & $5.1(4.9)$ & $5.5(4.7)$ & n.s. \\
\hline$\% \mathrm{CD}^{+}{ }^{+} \mathrm{T}$ cells, mean (SEM) & $38.9(4.1)$ & $38.1(1.9)$ & n.s. \\
\hline $\mathrm{CD}^{+}{ }^{+}$cell count nadir, mean (SEM) & $845.5(164.2)$ & $725.2(92.39)$ & n.s. \\
\hline CDC clinical stage $(\mathrm{N}-\mathrm{A} / \mathrm{B} / \mathrm{C})$ & $(6 / 0 / 0)$ & $(11 / 1 / 2)$ & - \\
\hline CDC immunological stage $(1 / 2 / 3)$ & $(4 / 2 / 0)$ & $(7 / 5 / 2)$ & - \\
\hline IGM (MG/DL), mean (SEM) & $92.9(12.6)$ & $97.5(18.6)$ & n.s. \\
\hline IGG (MG/DL), mean (SEM) & $980.3(76.5)$ & $869.8(183.2)$ & n.s. \\
\hline IGA (MG/DL), mean (SEM) & $147.7(19.3)$ & $106.5(7.4)$ & n.s. \\
\hline \multirow[t]{3}{*}{ ART therapy, N Patients (drugs) } & $2(2$ NRTIs $+1 \mathrm{Pl})$ & $7(2$ NRTIs $+1 \mathrm{PI})$ & n.s. \\
\hline & 4 (2 NRTIs + 1 NNRTI) & 4 (2 NRTIs + 1 NNRTI) & \\
\hline & & $3(2 \mathrm{NRTIS}+1 \mathrm{NNRTI}+1 \mathrm{PI})$ & \\
\hline
\end{tabular}

ART, antiretroviral therapy; AUC, area under the curve; CDC, Center for Disease Control and Prevention; Cl, confidence interval; n.s., not significant; ND, not determined; NNRTI, non-nucleoside reverse-transcriptase inhibitor; NRTI, nucleoside reverse-transcriptase inhibitor; PI, protease inhibitor; SEM, standard error of the mean. 
compared with seropositive study participants $(P=0.014$, $P=0.024, P=0.0001$ and 0.02 ) respectively; Table 1 and Supplementary Figure 3B, http://links.lww.com/QAD/ B649). Asterisks in Supplementary Figure 3 B, http:// links.lww.com/QAD/B649, indicate 3 seropositives who despite a similar exposition to seronegatives to the antigen, still maintain a positivity for HIV antigens. All patients have been treated according to PENTA guidelines [13] at the time of diagnosis irrespective of age.

\section{Gp140-specific B and T cells persist in both seronegative and seropositive early treated group after long-term viral suppression and higher IL21 production distinguishes HIV-specific T cells of seropositives}

To assess whether HIV serostatus was associated with differences in B-cell or T-cell phenotype, we investigated surface maturation markers of $\mathrm{B}$ and $\mathrm{T}$ cell by flow cytometry. Seropositive and seronegative study participants demonstrated similar B-cell compartment measured as frequency of $\operatorname{IgD}, \mathrm{CD} 27$, and CD21 as well as $\operatorname{IgG}$ and IgM among live $\mathrm{CD} 10^{-} \mathrm{CD} 19^{+} \mathrm{B}$ cells (Supplementary Figure $2 \mathrm{a}-\mathrm{d}$, http://links.lww.com/QAD/B648, as well as similar mature T-cell subsets and peripheral T-follicular helper cells (pTfH; live $\mathrm{CD}^{+} \mathrm{CD} 27^{+} \mathrm{CD} 45 \mathrm{RO}^{+}$ $\mathrm{CXCR}^{+}$) $($Supplementary Figure $4 \mathrm{a}-\mathrm{c}$, http://links.lww. com/QAD/B650, http://links.lww.com/QAD/B651).
We further investigated the frequency of gp140-specific B and $\mathrm{T}$ cells by flow cytometry. The presence of gp140specific $\mathrm{T}$ cells was quantified as $\mathrm{CD} 40 \mathrm{~L}^{+} \mathrm{T}$ cells following stimulation with gp140 and SEB [14] (representative gates in Fig. 1a). Seronegative and seropositive study participants were similar with respect to frequency of gp140-specific T cells (left panel, Fig. 1a). Characterization of the distribution of memory CD40L ${ }^{+}$ maturational T-cell subsets by expression of CD27, CD45RO (representative gate in Fig. 1b) and CXCR5 demonstrated that seronegative and seropositive study participants have similar gp140-specific T-cell distribution (Fig. 1c) and peripheral T-follicular helper cells (pTfh) (Fig. 1d). Both groups demonstrated a higher frequency of gp140-specific $\mathrm{T}$ cells within central memory $\mathrm{T}$ cells $\left(\mathrm{CD} 27^{+} \mathrm{CD} 45 \mathrm{RO}^{+}, \mathrm{CM}\right)$ compared with effector memory $\left(\mathrm{CD} 27-\mathrm{CD} 45 \mathrm{RO}^{+}, \mathrm{EM}\right)$ in both seronegative and seropositive individuals $(P=0.041$ and $P=0.004$, respectively; Fig. 1c). To further explore gp140-specific T-cell function, cytokine production was investigated by intracellular staining after gp140 protein stimulation. Whereas no differences were found for

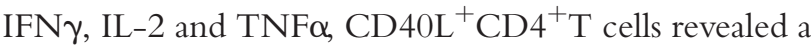
higher expression of IL-21 in seropositives compared to seronegatives $(P=0.003$; Fig. $2 \mathrm{a})$. We further evaluated the ability of gp140-specific T cells to produce cytokines simultaneously using Boolean analysis. Results confirmed the higher production of IL-21 by gp140-specific T cells

(a)

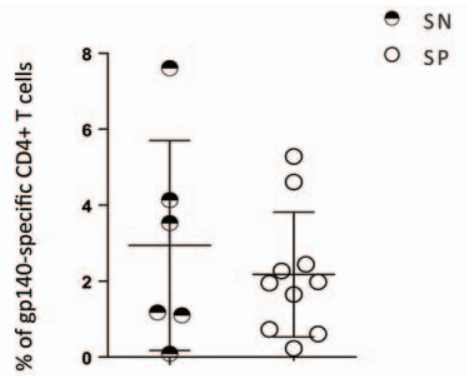

(b)

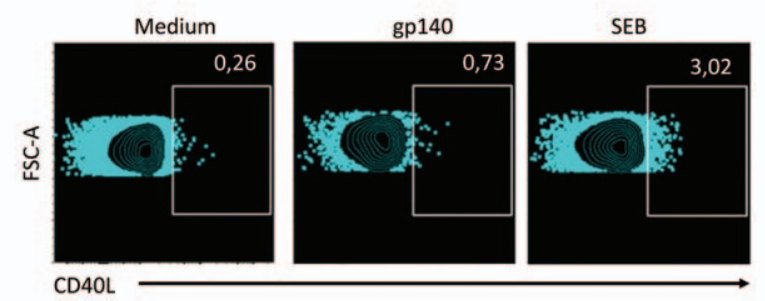

(c)

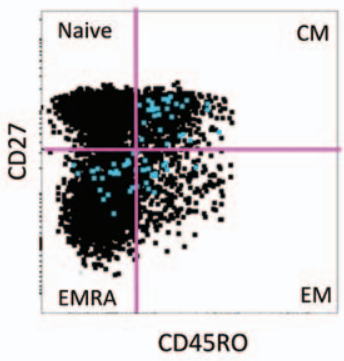

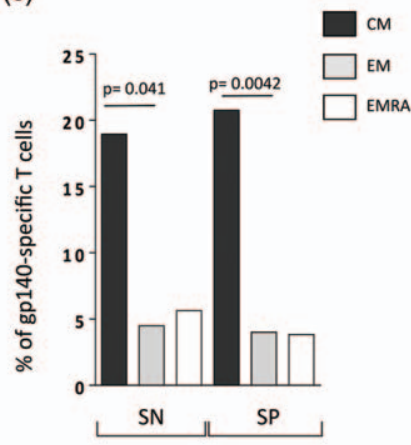

(d) Gated on live $\mathrm{CD} 3+\mathrm{CD} 4+\mathrm{CD} 4 \mathrm{OL}+\mathrm{CD} 27+\mathrm{CD} 45 \mathrm{RO}+$

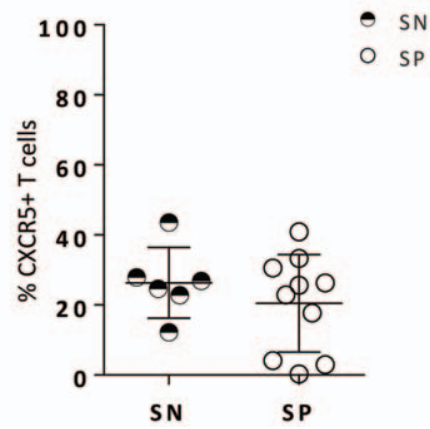

Fig. 1. Gp140-specific T cells. Left side panel of (a) shows a representative gate used to identify gp140-specific T-cell subsets according to the expression of CD40L after in-vitro stimulation. Right panel of (a) depicts frequencies of gp140-specific T-cellgated on live $\mathrm{CD}^{+}{ }^{+} \mathrm{CD} 4{ }^{+} \mathrm{T}$ cells. (b) Representative gate of gp140-specific T-cell distribution in CM, EM and EMRA. (c) Contingency plot representing the median values of gp140-specific T cells within T-cell subset. (d) Scatter dot plot shows gp140specific peripheral T-follicular cells. Mann-Whitney test was used for all comparisons. SEB, Staphylococcal Enterotoxin B. 
(a)
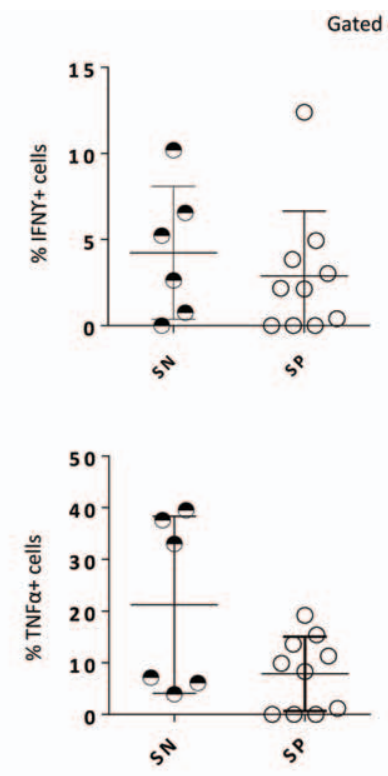

Gated on live $\mathrm{CD} 3+\mathrm{CD} 4+\mathrm{CD} 40 \mathrm{~L}+$
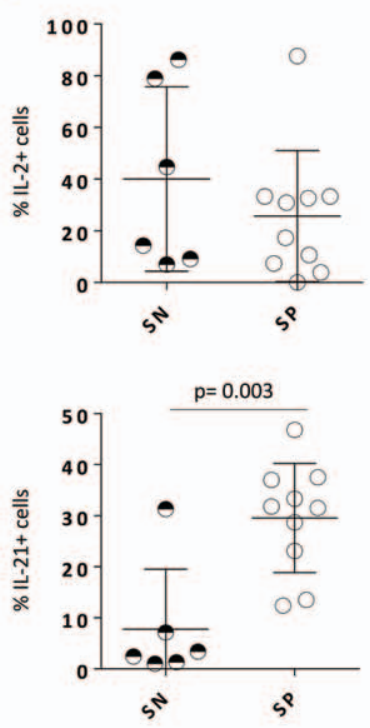

(b)

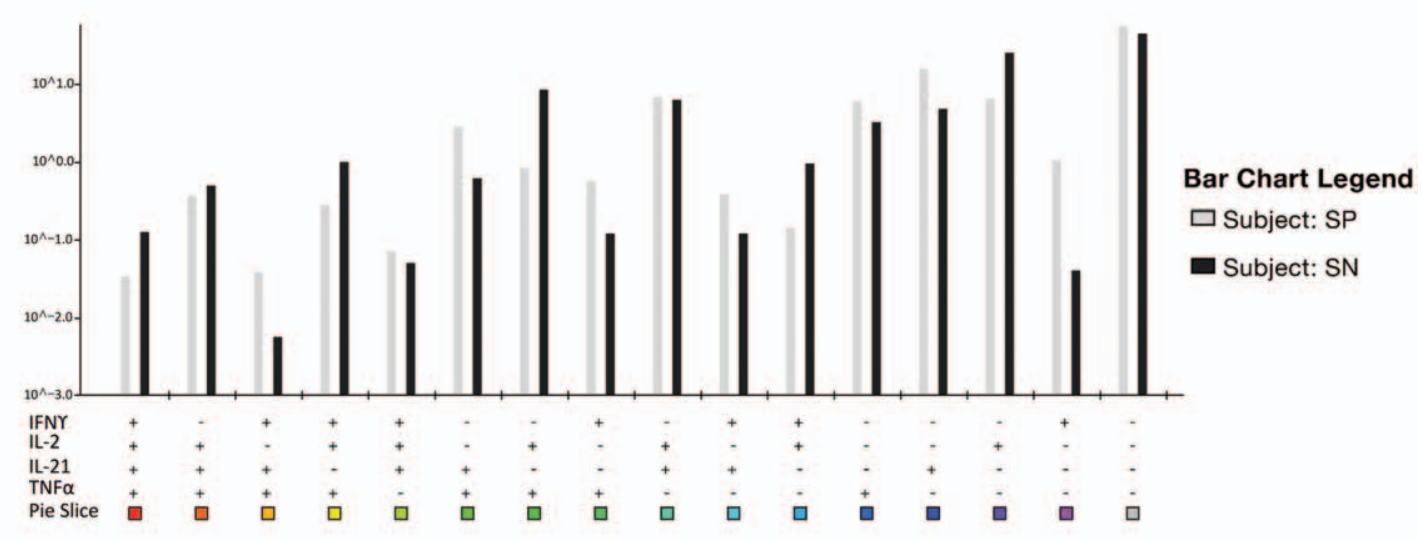

(c)

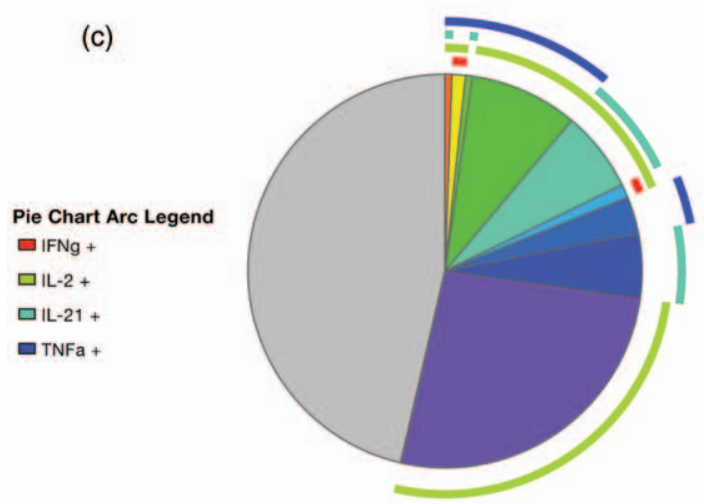

Subject: SN

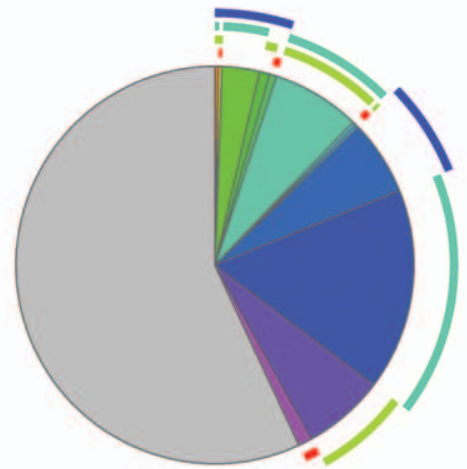

(d) $\bullet \mathrm{SN}$

O SP

Subject: SP

Fig. 2. Cytokine production in gp140-specific T cells. (a) Scatter dot plot representing intracellular staining measurment for IFN- $\gamma$, IL-2, TNF $\alpha$, IL-21 production after in-vitro stimulation in gp140-specific CD4 ${ }^{+} \mathrm{T}$ cells. (b) SPICE program was used for Boolean analysis looking at the production of IFN- $\gamma$, IL-2, IL-21 and TNF $\alpha$ in gp140-specific CD4 ${ }^{+}$T cells in seronegatives and SP. The bar graph represents the median frequency of all the Boolean subsets. (c) Each color in the pie charts corresponds to a specific combination of markers indicated at the bottom of the bar graph in (b), whereas every arc indicates the presence of that specific cytokine. (d) Permutation test was performed through SPICE program. Mann-Whitney test was used for comparisons $(* P \leq 0.05)$. 
(a)

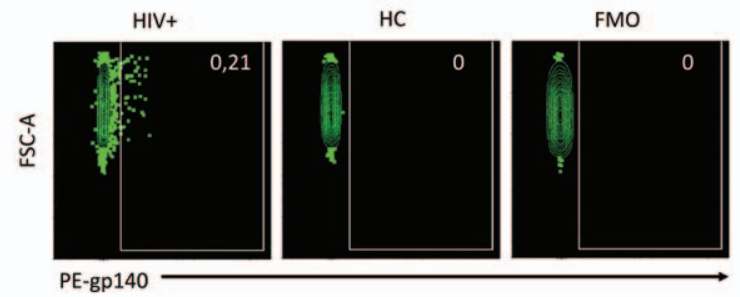

Gated on singlets, live CD19+ B cells

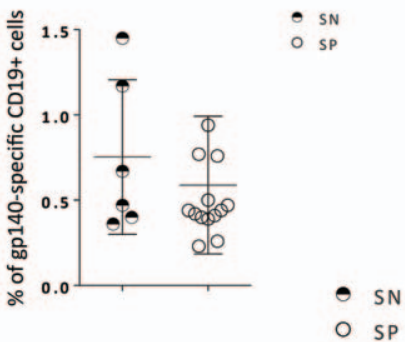

(b)
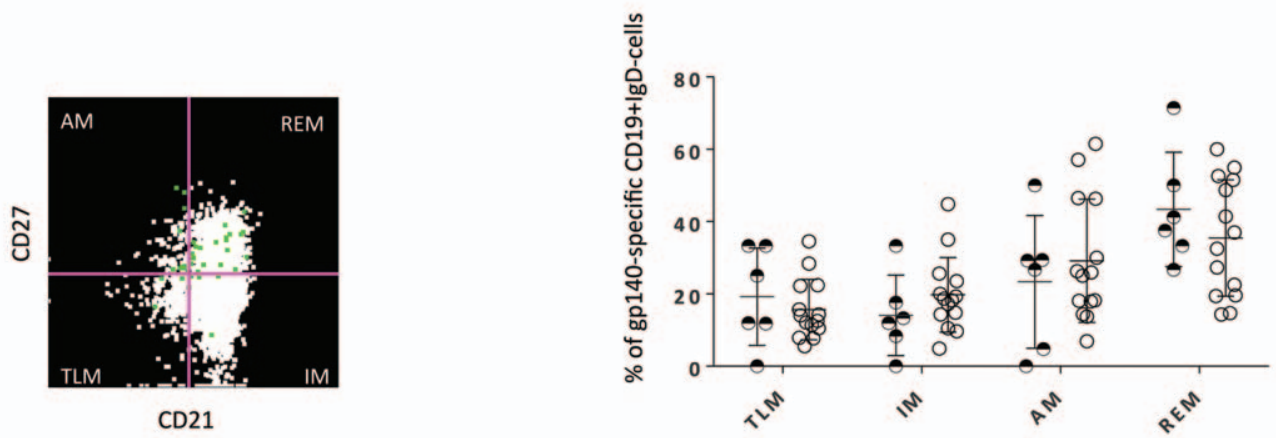

(c) Seronegative

(d) Seropositive
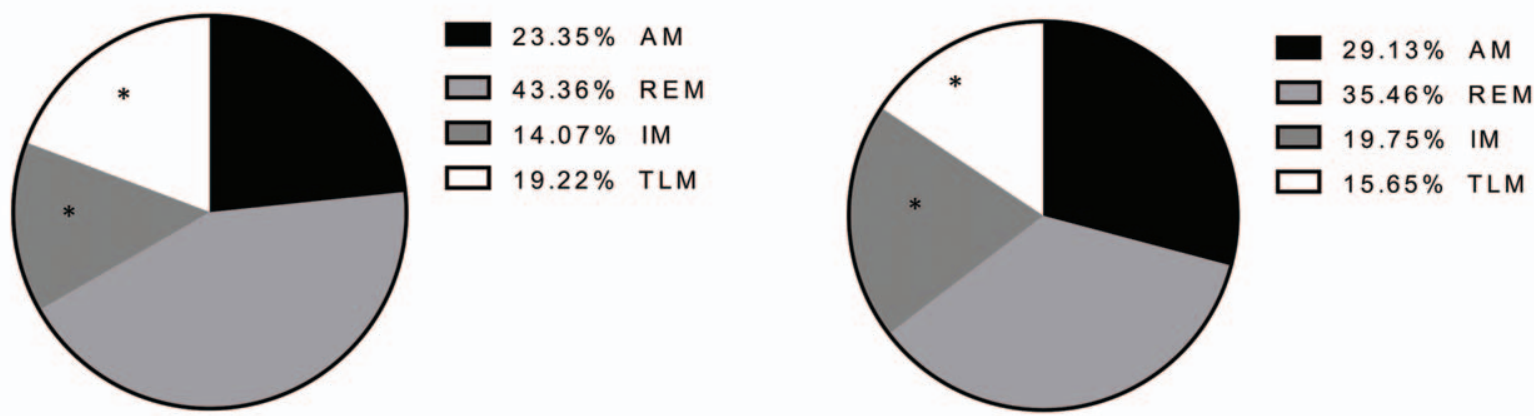

Fig. 3. Gp140-specific B cell distribution in total CD19 ${ }^{+}$cells and among maturational subsets. Left side panel in (a) depicts representative gates of gp140-specific staining in B cells with negative controls. Scatter dot plot on the right side of panel (a) represents the percentage of gp140-specific cells among total CD19 ${ }^{+}$B cells. (b) On the left side, demonstrative gate used to identify the surface expression of $\mathrm{CD} 21^{+}$and $\mathrm{CD} 27^{+}$on $\mathrm{CD} 19^{+} \mathrm{B}$ cells. On the right of panel (b), the scatter dot plot shows the frequencies of gp140-specific B cells among CD2 $7^{+}$and CD $21^{+}$subsets. Statistical analyses between the subsets were determined by Mann-Whitney test. (c and d) Median values of gp140-specific B-cell distribution among CD2 $7^{+}$and CD2 $1^{+}$subsets were used to build contingency plots showed in the pie charts. Unpaired $t$-test or Mann-Whitney U-test were used to compare normally or not-normally distributed data, respectively. AM, activated memory; FMO, fluorescence minus one; HC, healthy control; HIV+, HIV-positive patient; IM, intermediate memory; REM, resting memory; TLM, tissue-like memory. Asterisk (*) indicates statistical differences among REM cells and the other B cell populations.

from seropositive compared with seronegative study participants, and further revealed that most of these cells were IL-21 single producers (Fig. $2 b$ and c). Results of the permutation test confirm the difference present between the two populations (Fig. 2d).

Gp140-specific B cells were detected using a previously validated fluorochrome-conjugate trimeric gp140 protein probe (left panel Fig. 3a). Regardless of serostatus, both early treated groups presented comparable levels of gp140-specific B cells in total live CD19 ${ }^{+}$B cells (right panel Fig. 3a). In order to exclude low-affinity B-cell receptor (BCR) binding as previously shown [15-18], we further evaluated the gp140-specific IgD-B-cell subset among the maturational subsets and found no differences between seronegatives and seropositives (Fig. 3b). Gp140-specific B cell distribution were enriched within the resting memory $\left(\mathrm{CD} 27^{+} \mathrm{CD} 21^{+}\right.$, REM) subset compared with other subsets in both seronegatives $[P=0.006$ vs. immature memory and $P=0.015$ vs. Tissue Like Memory (TLM)] and seropositive patients $(P=0.005$ vs. immature memory; $P=0.0004$ vs. TLM; 
Fig. $3 c$ and d). These observations support our previously published study suggesting that early ART preserves an intact immune B-cell response in HIV-infected children [19].

\section{Early transcriptional signatures after HIV peptide stimulation distinguishes seropositives from seronegative early treated group}

After an overnight in-vitro stimulation with HIV gp140 peptides and CD40L, we assessed transcriptional profiles and changes in early programming [20] in switched $\operatorname{IgM}-\mathrm{IgD}-$ or in IgM+IgD- gp140-specific B cells. With such sorting strategy, we focused our analysis on high specificity gp140+ B cells avoiding the inclusion of low-affinity IgD + gp140-binding cells. We sort-purified fixed numbers (100) of gp140-specific B cells by flow cytometry and analyzed transcript levels in 96 previously selected and validated genes through the multiplex RT-PCR (Fluidigm; Biomark) (Supplementary Table 1, http://links.lww.com/QAD/B652). Basal levels of differentially expressed genes (DEGs) in switched IgM-IgD - B cells revealed similar profiles between seronegatives and seropositives with the exception of the SLAMF1 gene, encoding signaling lymphocytic activation molecule 1 (CD150), which was upregulated in seropositives vs. seronegatives (Fig. 4a). However, in gp140 peptide-stimulated samples, 13 DEGs were significantly upregulated in seropositives compared with seronegatives, including those involved in: signal transduction and activation after BCR/TLR triggering including MYD88, STAT3, LIGHT, MTOR, MAPK3, and SYK (Fig. 4a); (b) B-cell activation and proliferation after T-cell cognate stimuli, such as CD22 ${ }^{+}$and $\mathrm{CD} 86^{+}$, and (c) antibody production and class switch recombination (CSR) via active control of AICDA-, that is, BATF.

DEGs were also investigated in the IgM+IgD- gp140specific B-cell subset. In the unstimulated samples, seropositives showed upregulation of two DEGs, IL10 known to play a key role in the inhibitory B-cell activity and ABCB1, an ATP-dependent efflux pump, found as a discriminating factor of naive $\mathrm{B}$ cells [21]. Similar to what was found in stimulated $\mathrm{IgM}-\mathrm{IgD}-\mathrm{gp} 140$ specific B cells, the IgM+IgD- gp140-specific cells also presented a robust functional signature in seropositives compared with seronegatives. Indeed, 5 DEGs were found upregulated in seropositives compared with seronegatives in stimulated samples (Fig. 4a). PLC $\gamma$, mainly involved in $\mathrm{B}$-cell proliferation after BCR triggering, activation markers BATF and $\mathrm{CD} 38^{+}$, as well as CAMK4 and MZB1 downstream activators of antibody secretion through the control of $\mathrm{Ca}^{++}$stores were upregulated in seropositives compared with seronegatives.

In order to avoid interindividual gene expression variability, gene expression differences were also calculated according to paired differences between stimulated and unstimulated samples and defined as Differentially Induced Genes (DIGs) as previously shown [12]. Within the switched IgM-IgD- gp140specific $\mathrm{B}$ cells, seropositives demonstrated upregulation of IKBKG (NEMO), an NFKB activator, and IL2RA. A downregulation of the homing chemokine coreceptor CXCR 4 was found only in seronegatives group in both IgM - and IgM $+\operatorname{IgD}-$ gp140-specific B cells (Fig. 4b). Consistent with this finding, CXCR 4 was recently found to be downregulated in B cells of HIV-infected patients with lower serologic responses upon HBV vaccine [22]. Also, the IgM+IgD- gp140-specific counterpart showed similar results, indeed PRDM1 (BLIMP1) was positively induced only in seropositives, suggesting reduced plasma cell differentiation in IgM+ gp140-specific B cells in seronegative patients. Moreover, only seropositives had upregulation of genes involved in immune activation and lymphocyte proliferation pathways, such as CAMK4, CAV1, IL-21R. PDCD1 (PD-1), a regulator of B-cell activation [23], and SLAMF1 a regulator of antibody production [24] were downregulated only in seropositive patients (Fig. 4b).

\section{HIV antibody response and higher activation pattern in gp140-specific B-cell gene signature is associated with HIV-specific T-cell function (IL-21)}

To explore the most important variables defining humoral, and cellular responses upon HIV in early treated group, we performed a pairwise correlation matrix between gp140-specific T cells, gene expression of gp140-specific B cells and clinical data (Fig. 4c). A strong positive correlation was found between magnitude of antibody response, age at ART initiation and gene expression within the switched IgM-IgD- gp140specific B cells with 10 and 9 positively correlated genes, respectively (Fig. 4c).

Analysis further revealed a positive correlation between IL-21-producing gp140-specific $\mathrm{T}$ cells and gene expression within the stimulated switched IgM-IgDgp140-specific B-cell subsets. Indeed, 12 genes, including CCR7, CD22, CD86, CXCR5, MTOR and TLR7 positively correlated with IL-21-producing gp140specific $\mathrm{T}$ cells. A positive correlation was also present between the magnitude of HIV antibody and PRDM1 gene expression after in-vitro stimulation further suggesting the greater ability of gp140-specific B cells of seropositives to undergo plasma cell differentiation after in-vitro stimulation. The frequency of gp140specific B cells was negatively correlated with NKRF gene expression within the switched IgM-IgD- gp140specific B cells further suggesting that NKRF, a negative regulator of NFKB, even in the context of in vitroprovided gp140 peptides, may impact on gp140-specific B cells over time. 
(a)
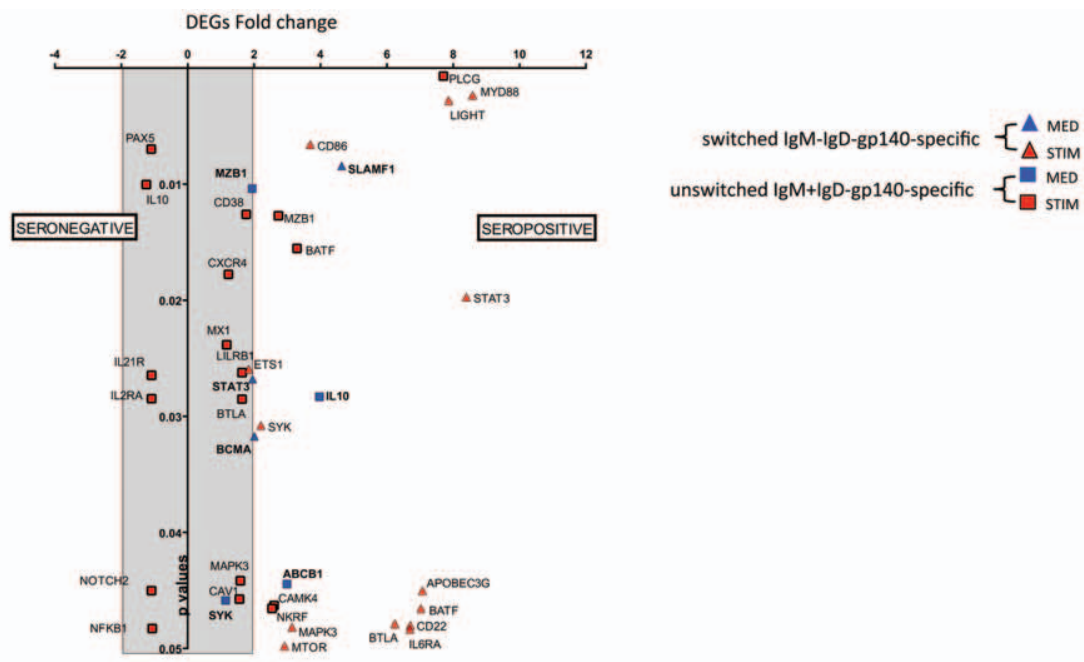

(b)
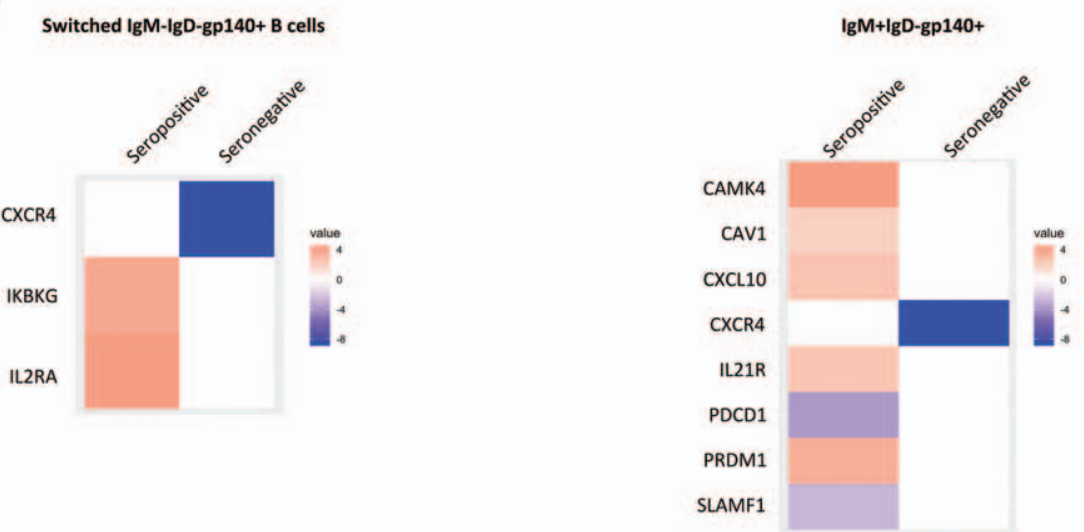

(c)

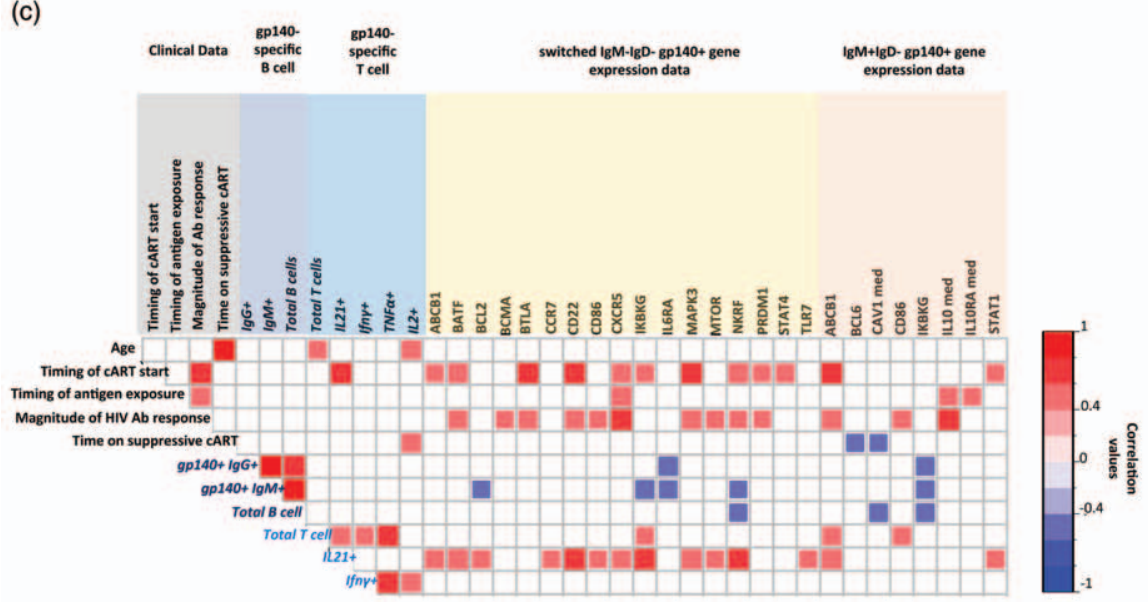

Fig. 4. Gene expression analysis in gp140-specific B-cell subsets. (a) Volcano plot representing DEGs between seropositive and seronegative, with (red dots) or without (blue dots) in-vitro stimulation in sorted IgM-gp140-specific (triangle dots) and IgM+gp140specific B cells (square dots). Only DEGs with a $P$ value less than 0.05 are shown in the volcano plot. (b) Column heatmaps demonstrate differentially induced genes (DIGs) between stimulated and unstimulated samples in IgM- gp140-specific B cells (left side panel) and IgM+gp140-specific B cells (right side panel). Values depicted in this panel were calculated by the difference in the averages in Et between the two experimental conditions for both groups and displayed in the heatmap. Only genes resulted significantly induced with a $P$ value less than 0.05 at paired analysis are showed in the heatmap. Paired Wilcoxon test for not-normally distributed data and paired $t$-test for normally distributed data were used for comparisons. (c) A correlation matrix is depicted with color heatmap showing $r$ values derived from Spearman and Pearson correlation analysis, respectively used for normally and notnormally distributed data. A color was assigned only to $r$ values resulting from significant correlations between data with a $P$ value less than 0.05. An $r$ value of 0 (white) was assigned to $P$ value greater than 0.05 . DEGs, differentially expressed genes. 


\section{Discussion}

The generation of an antigen-specific memory cell pool is a dynamic process that evolves from naive cells to a memory cell pool able to rapidly react upon antigenic restimulation. In perinatally HIV-infected children treated with ART early after birth with sustained long-term viral suppression, this process is influenced by both the limited duration of antigen exposure after birth, and the distinct neonatal immune system $[8,25]$. HIV-specific responses in such early treated children appears to be low or absent $[4,6]$, and this may be the result of a premature interruption of memory development. In line with previous reports [7,26,27], in our cohort, $30 \%$ of perinatally HIV-infected children treated within the first year of life resulted in seronegative status, and as expected, this sub-group was characterized by an earlier timing of ART start, lower antigen exposure and lower HIV-DNA levels compared with seropositives [7]. However, despite the rapid control of the plasma viremia compared with the other seropositive participants, three study participants were still positive for HIV-specific antibodies. Thus, it is not clear whether additional intrinsic characteristics other than viral exposure, may impact on HIV serostatus. It is also important to mention that, albeit not statistically significant, all but one of the seronegative patients are female patients. According to this data, and a recently published work [28] where 10 out of 11 posttreatment controllers were girls, we believe that sex may play a role in the ability to control HIV in early treated perinatally HIV-infected children. This aspect should be further investigated in larger studies. Indeed, one of the main limitation of the present study is the paucity of HIV Abnegative patients analyzed. However, HIV seronegative status in children growing with HIV is a rare event where early treatment initiation ( $<3$ months of age) followed by a continuous viral suppression together with still unknown host-related factors are necessary. In pediatric patients, such conditions, are critically limited by both adherence and compliance issues experienced in specific age ranges [29-31].

Regardless serological status, in the present study, we described that HIV-specific B and T cells persist at similar levels in both seronegative and seropositive groups of early treated children. Gp140-specific B cells were further investigated for their B-cell phenotype characteristics and showed that in both seronegatives and seropositives, gp140-specific IgD-B cells were found to be enriched in REM B cells (gp140-specific REM: 35.46 and $43.36 \%$ in seropositives and seronegatives, respectively) (Fig. 3) rather than activated subsets (AM and TLM B cells) $[19,32]$. As recently reported, this was not the case in ART-naive HIV-infected adults during the acute phase of the infection where gp140-specific B-cell responses were dominated by AM and TLM subsets rather than REM [15]. Overall, these results are consistent with our previous reports, demonstrating that early treatment in perinatally HIV-infected children is able to preserve the memory B-cell compartment preventing B-cell exhaustion, commonly found in chronically infected patients and late treated patients $[19,32]$.

To further evaluate whether qualitative differences within the gp140-specific B-cell subsets may underline the discordant ability of these patients to maintain HIV antibody over time, the transcriptional characteristics of switched IgM-IgD- gp140-specific B cells and unswitched IgM+IgD- gp140-specific B cells were further evaluated in two different ex-vivo conditions in seronegatives compared with seropositives. Regardless serostatus, no major differences emerged in terms of DEGs between the two groups in unstimulated conditions. However, switched IgM-IgD- gp140-specific B cells, presented a different pattern of activation after an overnight stimulation with HIV peptides and CD40L. Indeed 13 genes mainly involved in intracellular signal transduction (MYD88, STAT3, LIGHT, MTOR), B-cell activation (CD86, CD38, BATF and $S Y K)$ as well as in CSR $(B A T F)$ were upregulated in seropositives compared with seronegatives overall showing a different gp140-specific activation signature in these B cells. These data were further analyzed by paired analysis to exclude inter-individual gene expression variability. We showed that CXCR4, a homing receptor, both in switched IgM-IgD- and IgM+IgD- gp140-specific B cells, is negatively induced after in-vitro stimulation only in seronegative patients. These results are in line with recent data showing defective CXCR4 expression in HIVinfected patients demonstrating suboptimal responses upon $\mathrm{HBV}$ vaccination [22]. Other vaccine-induced $\mathrm{Ab}$ responses were evaluated in order to exclude intrinsic defect of $\mathrm{Ab}$ production in seronegatives. The analysis of pneumococcus $A b$ showed seroprotective titers for the majority of early treated with higher levels in seronegatives compared with seropositives (data not shown). These data suggest, as previosuly shown [19,33], that early treated patients and especially seronegatives present a preserved $\mathrm{Ab}$ response and $\mathrm{B}$-cell function compared with late-treated ones. In line with this, lack of HIVspecific $\mathrm{Ab}$ in seronegatives, may be because of a suppression of viral replication during a period of relative immunological immaturity rather than a B-cell intrinsic dysfunction [34]. A correlation matrix further revealed that the expression of PRDM1 in switched IgM-IgDgp140-specific B cells, necessary for the formation of mature $\mathrm{Ab}$-secreting plasma cells $[35,36]$, was positively associated to the magnitude of HIV Ab.

Overall, our observations suggest that despite similar frequency of gp140-specific B cells, qualitative differences may drive discordant ability of early treated patients to maintain antibody vs. HIV over time. The differential pattern showed by these cell subsets may be the result of a shorter antigen exposure in patients achieving a very early and sustained viral suppression in turn reducing cycles 
within the germinal centers and provide a truncated maturation process of gp140-specific B and T cells [34].

We further evaluated the T-cell compartment in order to dissect whether T-cell specific differences in both antigen-specific and general subsets may influence the T-cell dependent ability of early treated to maintain HIV antibody over time. Whereas no differences were found in terms of total gp140-specific T cell and memory subsets distribution, IL-21 production after in-vitro stimulation was higher in seropositives compared with seronegatives suggesting that a longer viral exposure in seropositives allowed a more complete maturation process of these cells. These results were further confirmed by the correlation matrix showing strong correlation of IL-21 production and early transcriptional signatures of switched gp140-specific B cells after stimulation. IL-21 plays a critical role in B-cell fate decisions and differentiation and influences the expression of several functionally important B-cell surface molecules [37]. We here show for the first time in the context of pediatric HIV-infected children treated early after birth and under viral control, that lower production of IL-21 from gp140specific $\mathrm{T}$ cells and concomitant lower gene expression after HIV peptide stimulation in vitro distinguish early treated seronegative children who fail to preserve HIV humoral immunity. Whereas these data particularly highlight the T-cell-dependent antibody response, additional experiments are needed in order to dissect the T-cell independent function of gp140-specific memory B cells in early treated patients.

Collectively, our data suggest that in seronegatives children undergoing eART, B cells and T cells are characterized by a reduced training process of maturation because of shorter antigen exposure, which provide qualitative differences both in terms of cytokine production and gene expression when the HIV antigen is re-encountered. In turn, this reduced training may hamper the ability of B cells to induce plasma cell differentiation upon re-stimulation and defective T/B cognate stimuli (e.g. IL-21) in the T-cell counterpart. It is still not clear, however, whether additional host-related characteristics, not solely driven by antigen exposure and the timing of ART initiation may impact the formation of a mature HIV-specific B cells able to induce and maintain HIV antibody in early treated individuals. Future studies with comprehensive multiOMIC systems biology approaches may further define such characteristics thereby informing personalized new disease-modifying interventions.

Our observations suggest that a truncated memory process [38] could occur because of early control of HIV replication after early treatment initiation, visualized in Supplementary Figure 1, http://links.lww.com/QAD/ B647. This finding is in line with other models of early and rapidly suppressed infection [39] with limited geminal center (GC) reactions that may be dominated by $\operatorname{IgM}+$ memory B cells rather than $\operatorname{IgG}[38$ ]. In the present study, although no difference was found in frequencies of $\mathrm{IgM}+\mathrm{IgD}-\mathrm{gp} 140-$ specific B cells between seronegatives and seropositives, positive induction of PRDM1 (Blimp1), IL21R, CAV1, and CAMK4 only in seropositives supports the ability of this B-cell subset to undergo a plasma cell differentiation upon restimulation. In addition, seronegative children, who failed to positively induce such genes, showed a negative induction of CXCR 4. Although the role and function of IgM+ memory B cells is still extensively debated [40,41], recent studies revealed greater transcriptional analogies with classic IgG+ rather than naive B cells. Indeed, IgM+ memory subset demonstrated evidence of SHM and ability to provide rapid humoral response upon antigen re-challenge in a murine malaria model $[39,42]$. Additional studies are needed in order to fully characterize such responses and to define possible novel targets of immune interventions aiming at inducing longterm effective HIV immune responses.

In conclusion, although gp140-specific T-cell and B-cell immunity persists in perinatally HIV-infected children treated early after birth with discordant HIV antibody profile after several years of HIV viral control, responses to antigen re-stimulation differ markedly with respect to early transcriptional signatures and function. Albeit limited by the small sample size, we believe that our observation may further inform future studies aiming at viral remission through immunization strategies targeting specific molecular pathways in early treated children with discordant HIV serologic profiles.

\section{Acknowledgements}

We acknowledge all patients and guardians who decided to participate to the study. We thank Emma Manno, Sonia Zicari, Alessandra Ruggiero, Libera Sessa, Nadia Iavarone, Tamara Di Marco for clinical and laboratory assistance and Daniel Gomezpena, Silvia Faggion, Jennifer Faudella and Inger Lindfors Rossi for administrative assistance. We further thank the Flow Cytometry Core Facility of the University of Miami.

Author contributions: P.P., P.R., N.C. and E.M., conceived the study and designed the experiments; N.C., E.M., Sa.R., St.R., I.P. and S.D.C. accomplished the experimental procedures and S.P. and P.P. provided supervision; N.C. and E.M. prepared the first version of the manuscript; N.C., P.P., S.B. and P.Z. provided clinical data; N.C., E.M., Sa.R., St.R., I.P. performed the formal analysis; all authors participated in writing, reviewing and editing the article; all authors and all the EPIICAL study team members provided supervision, contributed to scientific discussion and supported the article; S.P., P.R., and P.P. provided support and resources. 
Funding: This work is part of the EPIICAL project (www.epiical.org), supported by PENTA-ID foundation (www.penta-id.org) and by an independent ViiV Healthcare UK grant. P.P. was also supported by AI12734704. S.P. was supported by two grants (AI108472 and AI127347). Work performed at the Laboratory Sciences Core of the Miami was supported by CFAR (P30AI073961) and by the following NIH Co-Funding and Participating Institutes and Centres: NIAID, NCI, NICHD, NHLBI, NIDA, NIMH, NIA, NIDDK, NIGMS, FIC, and OAR. Additional support was obtained by grants from Children's Hospital Bambino Gesú (Ricerca corrente 2016), and Associazione Volontari Bambino Gesù. $\mathrm{OL}$ and the Precision Vaccines Program are supported in part by an National Institute of Health/National Institute of Allergy and Infectious Diseases Division of AIDS administrative supplement to Human Immunology Project Consortium U19AI118608 as well as by the Department of Pediatrics, Boston Children's Hospital.

\section{Members of the EPIICAL consortium}

Nigel Klein, Diana Gibb, Sarah Watters, Man Chan, Laura McCoy, Abdel Babiker, Anne-Genevieve Marcelin, Vincent Calvez, Maria Angeles Munoz, Britta Wahren, Caroline Foster, Mark Cotton, Merlin Robb, Jintanat Ananworanich, Polly Claiden, Deenan Pillay, Deborah Persaud, Rob J De Boer, Juliane Schröter, Anet J N Anelone, Thanyawee Puthanakit, Adriana Ceci, Viviana Giannuzzi, Kathrine Luzuriaga, Nicolas Chomont, Mark Cameron, Caterina Cancrini, Andrew Yates, Louise Kuhn, Avy Violari, Kennedy Otwombe, Ilaria Pepponi, Francesca Rocchi, Alfredo Tagarro, Maria Grazia Lain, Paula Vaz, Elisa Lopez, Tacita Nhampossa, Carlo Giaquinto, Anita De Rossi, Eleni Nastouli.

\section{Conflicts of interest}

There are no conflicts of interest.

\section{References}

1. Shiau S, Abrams EJ, Arpadi SM, Kuhn L. Early antiretroviral therapy in HIV-infected infants: can it lead to HIV remission? Lancet HIV 2018; 5:e250-e258.

2. Luzuriaga K. Early combination antiretroviral therapy limits HIV1 persistence in children. Annu Rev Med 2016; 67:201-213.

3. Ananworanich J, Puthanakit T, Suntarattiwong P, Chokephaibulkit K, Kerr SJ, Fromentin R, et al., HIV-NAT 194 Study Group. Reduced markers of HIV persistence and restricted HIV-specific immune responses after early antiretroviral therapy in children. AIDS 2014; 28:1015-1020.

4. Luzuriaga K, McManus M, Catalina M, Mayack S, Sharkey M, Stevenson M, Sullivan JL. Early therapy of vertical human immunodeficiency virus type 1 (HIV-1) infection: control of viral replication and absence of persistent HIV-1-specific immune responses. J Virol 2000; 74:6984-6991.

5. Persaud D, Patel K, Karalius B, Rainwater-Lovett K, Ziemniak C, Ellis A, et al., Pediatric HIV/AIDS Cohort Study. Influence of age at virologic control on peripheral blood human immunodeficiency virus reservoir size and serostatus in perinatally infected adolescents. JAMA Pediatr 2014; 168:1138-1146.
6. Kuhn L, Paximadis M, Da Costa Dias B, Loubser S, Strehlau R, Patel $F$, et al. Age at antiretroviral therapy initiation and cellassociated HIV-1 DNA levels in HIV-1-infected children. PLOS One 2018; 13:e0195514.

7. Rocca S, Zangari P, Cotugno N, De Rossi A, Ferns B, Petricone D, et al., EPIICAL Consortium. Human immunodeficiency virus (HIV)-antibody repertoire estimates reservoir size and time of antiretroviral therapy initiation in virally suppressed perinatally HIV-infected children. J Pediatric Infect Dis Soc 2018; 8:433-438.

8. Klein N, Palma P, Luzuriaga K, Pahwa S, Nastouli E, Gibb DM, et al. Early antiretroviral therapy in children perinatally infected with HIV: a unique opportunity to implement immunotherapeutic approaches to prolong viral remission. Lancet Infect Dis 2015; 15:1108-1114.

9. Rainwater-Lovett K, Uprety P, Persaud D. Advances and hope for perinatal HIV remission and cure in children and adolescents. Curr Opin Pediatr 2016; 28:86-92.

10. Guan $Y$, Sajadi MM, Kamin-Lewis R, Fouts TR, Dimitrov A, Zhang $Z$, et al. Discordant memory B cell and circulating antiEnv antibody responses in HIV-1 infection. Proc Natl Acad Sci U S A 2009; 106:3952-3957.

11. Cotugno N, De Armas L, Pallikkuth S, Rinaldi S, Issac B, Cagigi $A$, et al. Perturbation of B cell gene expression persists in HIV infected children despite effective antiretroviral therapy and predicts H1N1 response. Front Immunol 2017; 8:1083.

12. de Armas LR, Cotugno N, Pallikkuth S, Pan L, Rinaldi S, Sanchez MC, et al. Induction of IL21 in peripheral T follicular helper cells is an indicator of influenza vaccine response in a previously vaccinated HIV-infected pediatric cohort. I Immunol 2017; 198:1995-2005.

13. Foster C, Bamford A, Turkova A, Welch S, Klein N, Group PGW, et al. Paediatric European Network for Treatment of AIDS Treatment Guideline 2016 update: antiretroviral therapy recommended for all children living with HIV. HIV Med 2017; 18:133-134.

14. Chattopadhyay PK, Yu J, Roederer M. Live-cell assay to detect antigen-specific CD4+ T-cell responses by CD154 expression. Nat Protoc 2006; 1:1-6.

15. Kardava L, Moir S, Shah N, Wang W, Wilson R, Buckner CM, et al. Abnormal B cell memory subsets dominate HIV-specific responses in infected individuals. J Clin Invest 2014; 124:3252-3262.

16. Haas A, Zimmermann K, Oxenius A. Antigen-dependent and independent mechanisms of $\mathrm{T}$ and $\mathrm{B}$ cell hyperactivation during chronic HIV-1 infection. J Virol 2011; 85:12102-12113.

17. Jelicic K, Cimbro R, Nawaz F, Huang da W, Zheng X, Yang J, et al. The HIV-1 envelope protein gp120 impairs B cell proliferation by inducing TGF-beta1 production and FCRL4 expression. Nat Immunol 2013; 14:1256-1265.

18. Brooks JF, Liu X, Davies JM, Wells JW, Steptoe RJ. Tetramerbased identification of naive antigen-specific $B$ cells within a polyclonal repertoire. Eur J Immunol 2018; 48:1251-1254.

19. Pensieroso S, Cagigi A, Palma P, Nilsson A, Capponi C, Freda E, et al. Timing of HAART defines the integrity of memory $B$ cells and the longevity of humoral responses in HIV-1 vertically-infected children. Proc Natl Acad Sci U S A 2009; 106:7939-7944.

20. McHeyzer-Williams LJ, Milpied PJ, Okitsu SL, McHeyzer-Williams MG. Class-switched memory B cells remodel BCRs within secondary germinal centers. Nat Immunol 2015; 16:296-305.

21. Wirths S, Lanzavecchia A. ABCB1 transporter discriminates human resting naive $B$ cells from cycling transitional and memory B cells. Eur J Immunol 2005; 35:3433-3441.

22. Bekele Y, Lemma M, Bobosha K, Yibeltal D, Nasi A, Gebre M, et al. Homing defects of B cells in HIV-1 infected children impair vaccination responses. Vaccine 2019; 37:2348-2355.

23. Thibult ML, Mamessier E, Gertner-Dardenne J, Pastor S, JustLandi S, Xerri L, et al. PD-1 is a novel regulator of human B-cell activation. Int Immunol 2013; 25:129-137.

24. Wang N, Halibozek PJ, Yigit B, Zhao H, O'Keeffe MS, Sage P, Terhorst C. Negative regulation of humoral immunity due to interplay between the SLAMF1, SLAMF5, and SLAMF6 receptors. Front Immunol 2015; 6:158.

25. Cotton MF, Violari A, Otwombe K, Panchia R, Dobbels E, Rabie $\mathrm{H}$, et al., CHER Study Team. Early time-limited antiretroviral therapy versus deferred therapy in South African infants infected with HIV: results from the children with HIV early antiretroviral (CHER) randomised trial. Lancet 2013; 382:1555-1563. 
26. McManus M, Henderson J, Gautam A, Brody R, Weiss ER, Persaud D, et al., PACTG 356 Investigators. Quantitative human immunodeficiency virus (HIV)-1 antibodies correlate with plasma HIV-1 RNA and cell-associated DNA levels in children on antiretroviral therapy. Clin Infect Dis 2018; 68:1725-1732.

27. Payne H, Mkhize N, Otwombe K, Lewis J, Panchia R, Callard R, et al. Reactivity of routine HIV antibody tests in children who initiated antiretroviral therapy in early infancy as part of the Children with HIV Early Antiretroviral Therapy (CHER) trial: a retrospective analysis. Lancet Infect Dis 2015; 15:803-809.

28. Vieira VA, Zuidewind P, Muenchhoff M, Roider J, Millar J, Clapson $M$, et al. Strong sex bias in elite control of paediatric HIV infection. AIDS 2019; 33:67-75.

29. Nachega JB, Uthman OA, Anderson J, Peltzer K, Wampold S, Cotton MF, et al. Adherence to antiretroviral therapy during and after pregnancy in low-income, middle-income, and highincome countries: a systematic review and meta-analysis. AIDS 2012; 26:2039-2052.

30. Schlatter AF, Deathe AR, Vreeman RC. The need for pediatric formulations to treat children with HIV. AIDS Res Treat 2016; 2016:1654938.

31. Giannattasio A, Albano F, Giacomet V, Guarino A. The changing pattern of adherence to antiretroviral therapy assessed at two time points, 12 months apart, in a cohort of HIV-infected children. Expert Opin Pharmacother 2009; 10:2773-2778.

32. Muir R, Metcalf T, Tardif V, Takata H, Phanuphak N, Kroon E, et al., RV254/SEARCH010 RV304/SEARCH 013 Study Groups. Altered memory circulating $\mathrm{T}$ follicular helper-B cell interaction in early acute HIV infection. PLoS Pathog 2016; 12:e1005777.

33. Cagigi A, Rinaldi S, Cotugno N, Manno EC, Santilli V, Mora N, et al. Early highly active antiretroviral therapy enhances B-cell longevity: a 5 year follow up. Pediatr Infect Dis J 2014; 33:e126-e131.
34. Palma P, Romiti ML, Cancrini C, Pensieroso S, Montesano C, Bernardi $S$, et al. Delayed early antiretroviral treatment is associated with an HIV-specific long-term cellular response in HIV-1 vertically infected infants. Vaccine 2008; 26:51965201.

35. Minnich $\mathrm{M}$, Tagoh $\mathrm{H}$, Bonelt $\mathrm{P}$, Axelsson $\mathrm{E}$, Fischer $\mathrm{M}$, Cebolla $B$, et al. Multifunctional role of the transcription factor Blimp-1 in coordinating plasma cell differentiation. Nat Immunol 2016; 17:331-343.

36. Higgins BW, McHeyzer-Williams LJ, McHeyzer-Williams MG Programming isotype-specific plasma cell function. Trends Immunol 2019; 40:345-357.

37. Pallikkuth S, Pahwa S. Interleukin-21 and $\mathbf{T}$ follicular helper cells in HIV infection: research focus and future perspectives. Immunol Res 2013; 57:279-291.

38. Seifert M, Kuppers R. Human memory B cells. Leukemia 2016; 30:2283-2292.

39. Krishnamurty AT, Thouvenel CD, Portugal S, Keitany GJ, Kim KS, Holder A, et al. Somatically hypermutated plasmodium-specific $\operatorname{IgM}(+)$ memory B cells are rapid, plastic, early responders upon malaria rechallenge. Immunity 2016; 45:402-414.

40. Seifert M, Przekopowitz M, Taudien S, Lollies A, Ronge V, Drees B, et al. Functional capacities of human IgM memory B cells in early inflammatory responses and secondary germinal center reactions. Proc Natl Acad Sci U S A 2015; 112:E546E555.

41. Capolunghi F, Rosado MM, Sinibaldi M, Aranburu A, Carsetti R. Why do we need IgM memory B cells? Immunol Lett 2013; 152:114-120.

42. Ehrenstein MR, Notley CA. The importance of natural IgM: scavenger, protector and regulator. Nat Rev Immunol 2010; 10:778-786. 